\title{
On $F_{3}(k, n)$-numbers of the Fibonacci type
}

\author{
Engin Özkan ${ }^{1} \cdot$ Nur Şeyma Yilmaz ${ }^{2} \cdot$ Andrzej Włoch $^{3}$
}

Received: 13 May 2021 / Accepted: 18 August 2021 / Published online: 7 October 2021

(C) The Author(s) . This article is published with open access at Springerlink.com

\begin{abstract}
In this paper, we study a generalization of Narayana's numbers and Padovan's numbers. This generalization also includes a sequence whose elements are Fibonacci numbers repeated three times. We give combinatorial interpretations and a graph interpretation of these numbers. In addition, we examine matrix generators and determine connections with Pascal's triangle.
\end{abstract}

Keywords Generalized Fibonacci numbers · Narayana's numbers · Padovan's numbers · Generating function · Pascal's triangle

Mathematics Subject Classification 11B37 $\cdot$ 11B39 $\cdot$ 11B65

\section{Introduction}

Integer sequences have always attracted the attention of many researchers, as number sequences find application in many other fields of science as well as in mathematics. Therefore, many generalizations and polynomials of these generalizations have been given and their properties have been studied [4-6, 9-12, 16].

Andrzej Włoch

awloch@prz.edu.pl

Engin Özkan

eozkan@erzincan.edu.tr

Nur Şeyma Yilmaz

nurseyma_ciceksiz@hotmail.com

1 Department of Mathematics, Faculty of Arts and Sciences, Erzincan Binali Yıldırım University, Erzincan, Turkey

2 Graduate School of Natural and Applied Sciences, Erzincan Binali Yıldırım University, Erzincan, Turkey

3 The Faculty of Mathematics and Applied Physics, Rzeszow University of Technology, al. Powstańców Warszawy 8, 35-959 Rzeszow, Poland 
The best known integer sequence is undoubtedly the Fibonacci sequence. Fibonacci numbers $F_{n}$ are defined by the recurrence

$$
F_{n}=F_{n-1}+F_{n-2}, \text { for } n \geq 2
$$

with initial conditions, $F_{0}=0$ and $F_{1}=1$. Narayana's numbers and Padovan's numbers, defined below, are not so popular.

$$
\begin{aligned}
& N_{n}=N_{n-1}+N_{n-3} \text { for } n \geq 3, \text { with } N_{0}=0, N_{1}=N_{2}=1 \\
& P v(n)=P v(n-2)+P v(n-3) \text { for } n \geq 3, \text { with } P v(0)=P v(1)=P v(2)=1
\end{aligned}
$$

But sequences formed by these numbers have also interesting properties. In this paper, we study a generalized sequence which generalize sequence of Narayana's numbers and sequence of Padovan's numbers, simultaneously. Moreover, its special cases are sequences formed by other known numbers; Fibonacci numbers and powers of 2. The aim of this study is to present, for these sequences, combinatorial interpretations, some explicit formulas, generating functions, matrix generators and determine connections with the Pascal triangle.

First we recall two types of generalizations of Fibonacci numbers used in our considerations.

First type. For an arbitrary $k \geq 2$ the $n$th generalized Fibonacci number is a sum of $k$ terms. Such generalizations was studied among others by Miles [8], Er [3] end recently by Włoch and Włoch [16].

In [8], Miles defined $k$-Fibonacci numbers where

$$
g_{n}=g_{(n-1)}+g_{(n-2)}+\cdots+g_{(n-k)} \text {, for } n \geq k \geq 2
$$

with $g_{0}=g_{1}=\cdots=g_{(k-2)}=0$ and $g_{(k-1)}=1$. In [3] is introduced a family of $k$ sequences generalized Fibonacci numbers in the following way. Let $k \geq 2, c_{j}, j \in$ $\{1,2, \ldots, k\}$ be integers. Then for an integer $1 \leq i \leq k$, generalized Fibonacci numbers are defined as

$$
f_{n}^{i}=\sum_{j=1}^{k} c_{j} f_{n-j}^{i}, \text { for } n>0
$$

with initial conditions $f_{n}^{i}=\left\{\begin{array}{c}1 \quad \text { if } i=1-n, \\ 0 \quad \text { otherwise, }\end{array}\right.$ for $1-k \leq n \leq 0$.

In [16], it was studied Fibonacci type numbers defined recursively by the kth order linear recurrence relation.

Let $k \geq 2, c_{i} \geq 0, i \in\{1, \ldots, k\}$ be integers such that there are at least two positive integers $c_{p}, c_{q}$ where $p \neq q$ and $1 \leq p, q \leq k$.

$$
f_{n}=c_{1} f_{n-1}+c_{2} f_{n-2}+\cdots+c_{n} f_{n-k}, \text { for } n>0
$$

with given nonnegative integers $f_{1-k}, \ldots, f_{-1}, f_{0}$ and there is $1-k \leq j \leq 0$ such that $f_{j}>0$.

For special values of $k, c_{i}$ and $f_{1-i}, i \in\{1,2, \ldots, k\}$ the formula (3) gives the well-known classical sequences. 
Let $k \geq 2$ be an integer. Generally sequences defined recursively by the $k$ th order linear recurrence relation of the form

$$
a_{n}=b_{1} a_{n-1}+b_{2} a_{n-2}+\cdots+b_{n} a_{n-k}, \text { for } n \geq k
$$

$b_{i} \in N \cup\{0\}, i=1, \ldots, k$ with fixed integers $a_{0}, \ldots, a_{k-1}$ are named as sequences of the Fibonacci type.

The second type. For an arbitrary $k \geq 2$ the $n$th generalized Fibonacci number is a sum of two terms, the $(n-k)$-th term and the second chosen in such way that obtained recurrence generalizes (1). We recall some of such generalizations.

Kwaśnik and Włoch studied in [7] generalized Fibonacci numbers $F(k, n)$ defined recursively as follows:

$$
F(k, n)=F(k, n-1)+F(k, n-k), \text { for } n \geq k+1
$$

with $F(k, n)=n+1$, for $n \leq k$. These numbers have many interpretations in graph theory [1].

In [2], Bednarz et al. introduced a different kind of distance generalization of Fibonacci numbers. This numbers are showed by $F d(k, n)$ and defined recursively as follows:

$$
F d(k, n)=F d(k, n-k+1)+F d(k, n-k), \text { for } n \geq k \geq 2
$$

with initial conditions $F d(k, n)=1$ for $0 \leq n \leq k-1$.

One of the other works in this field is [15] where they introduced a new kind of distance Fibonacci numbers as follows:

$$
F_{2}(k, n)=F_{2}(k, n-2)+F_{2}(k, n-k), \text { for } n \geq k
$$

with initial conditions $F_{2}(k, i)=1$ for $i=0,1,2, \ldots, k-1$.

In this paper, we will study special cases of defined above Fibonacci type numbers. Let $k \geq 1, n \geq 0$ be integers. $(3, k)$-distance Fibonacci numbers are define by the following recurrence relation;

$$
F_{3}(k, n)=F_{3}(k, n-3)+F_{3}(k, n-k), \text { for } n \geq \max \{3, k\}
$$

with initial conditions $F_{3}(k, n)=1$, for $n=0,1,2,3 \ldots, \max \{2, k-1\}$.

Recurrence $(5)$ of $(3, k)$-distance Fibonacci numbers generalizes recurrences of Narayana's numbers and Padovan's numbers.

$F_{3}(1, n+1)=N_{n}$ sequence A000930 in OEIS [13].

$F_{3}(2, n)=P v(n)$ sequence A000931 in OEIS [13].

$F_{3}(3, n)=2^{\left\lfloor\frac{n}{3}\right\rfloor}$ contains powers of 2 repeated 3 times, sequence A173862 in OEIS [13].

$F_{3}(4, n)$ sequence A079398 in OEIS [13].

$F_{3}(5, n)$ sequence A226503 in OEIS [13].

$F_{3}(6, n)=F_{\left\lfloor\frac{n}{3}\right\rfloor}$ contains Fibonacci numbers repeated 3 times, sequence A247049 in OEIS [13].

A few initial elements of these sequences for special values of $k$ and $n$ are included in the Table 1. 
Table $1(3, k)$-distance Fibonacci numbers $F_{3}(k, n)$

\begin{tabular}{|c|c|c|c|c|c|c|c|c|c|c|}
\hline$n$ & 0 & 1 & & & 3 & 4 & 5 & 6 & 7 & 8 \\
\hline$F_{3}(1, n)$ & 1 & 1 & & & 2 & 3 & 4 & 6 & 9 & 13 \\
\hline$F_{3}(2, n)$ & 1 & 1 & & & 2 & 2 & 3 & 4 & 5 & 7 \\
\hline$F_{3}(3, n)$ & 1 & 1 & & & 2 & 2 & 2 & 4 & 4 & 4 \\
\hline$F_{3}(4, n)$ & 1 & 1 & & & 1 & 2 & 2 & 2 & 3 & 4 \\
\hline$F_{3}(5, n)$ & 1 & 1 & & & 1 & 1 & 2 & 2 & 2 & 3 \\
\hline$F_{3}(6, n)$ & 1 & 1 & & & 1 & 1 & 1 & 2 & 2 & 2 \\
\hline$F_{3}(7, n)$ & 1 & 1 & & & 1 & 1 & 1 & 1 & 2 & 2 \\
\hline$n$ & 9 & 10 & 11 & 12 & 13 & 14 & 15 & 16 & 17 & 18 \\
\hline$F_{3}(1, n)$ & 19 & 28 & 41 & 60 & 88 & 129 & 189 & 277 & 406 & 595 \\
\hline$F_{3}(2, n)$ & 9 & 12 & 16 & 21 & 28 & 37 & 49 & 65 & 86 & 114 \\
\hline$F_{3}(3, n)$ & 8 & 8 & 8 & 16 & 16 & 16 & 32 & 32 & 32 & 64 \\
\hline$F_{3}(4, n)$ & 4 & 5 & 7 & 8 & 9 & 12 & 15 & 17 & 21 & 27 \\
\hline$F_{3}(5, n)$ & 3 & 4 & 5 & 5 & 7 & 8 & 9 & 12 & 13 & 16 \\
\hline$F_{3}(6, n)$ & 3 & 3 & 3 & 5 & 5 & 5 & 8 & 8 & 8 & 13 \\
\hline$F_{3}(7, n)$ & 2 & 3 & 3 & 3 & 4 & 5 & 5 & 6 & 8 & 8 \\
\hline
\end{tabular}

For a given sequence $a(n)$, sequences $a\left(\left\lfloor\frac{n}{3}\right\rfloor\right)$ and $a\left(\left\lfloor\frac{n}{2}\right\rfloor\right)$, with repeated elements, we will call a tripled $a(n)$ sequence and a doubled $a(n)$ sequence, respectively.

It is known that tiling defined by the Fibonacci numbers cover a plane. In [15] it was shown a tiling covering of a plane by tiling defined by doubled Fibonacci sequence. We present a tiling covering of a plane by tripled Fibonacci sequence, see Fig. 1.

Similar to classical Fibonacci numbers, numbers $F_{3}(k, n)$ can be extended to negative integers. Let $k \geq 4$ be integer and $F_{3}(k, n)=1$ for $n=0,1, \ldots, k-1$.

$$
F_{3}(k,-n)=F_{3}(k,-n+k)-F_{3}(k,-n+(k-3)) .
$$

Moreover for $k=1,2,3$,

$$
\begin{aligned}
& F_{3}(1,-n)=F_{3}(1,-n+3)-F_{3}(1,-n+2), \\
& F_{3}(2,-n)=F_{3}(2,-n+3)-F_{3}(2,-n+1) \text { and }
\end{aligned}
$$

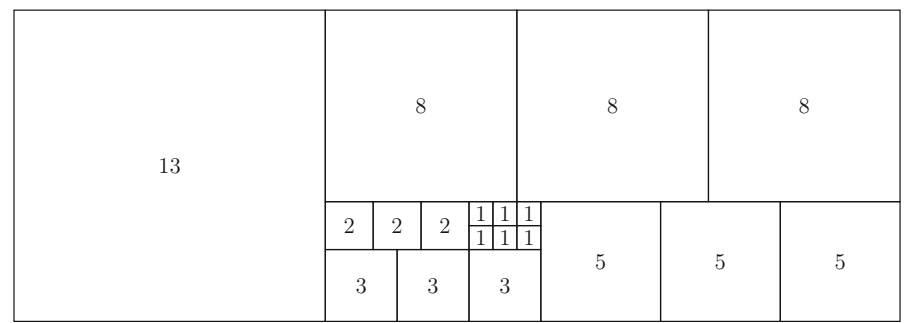

Fig. 1 A tiling interpretation of the tripled Fibonacci sequence $F_{3}(6, n)$ f 
Table 2 Numbers $F_{3}(k, n)$ for negative $n$

\begin{tabular}{|c|c|c|c|c|c|c|c|c|c|c|}
\hline$n$ & 18 & 17 & 16 & 15 & 14 & 13 & & 12 & 11 & 10 \\
\hline$F_{3}(1, n)$ & 4 & -8 & 1 & 5 & -3 & -2 & & 3 & 0 & -2 \\
\hline$F_{3}(2, n)$ & -5 & 4 & -3 & 1 & 1 & -2 & & 2 & -1 & 0 \\
\hline$F_{3}(3, n)$ & $\frac{1}{64}$ & $\frac{1}{32}$ & $\frac{1}{32}$ & $\frac{1}{32}$ & $\frac{1}{16}$ & $\frac{1}{16}$ & & $\frac{1}{16}$ & $\frac{1}{8}$ & $\frac{1}{8}$ \\
\hline$F_{3}(4, n)$ & -29 & 21 & -15 & 11 & -8 & 6 & & -4 & 3 & -2 \\
\hline$F_{3}(5, n)$ & -1 & 4 & 2 & -2 & -2 & 1 & & 2 & 0 & -1 \\
\hline$F_{3}(6, n)$ & -3 & 2 & 2 & 2 & -1 & -1 & & -1 & 1 & 1 \\
\hline$F_{3}(7, n)$ & 1 & -1 & -1 & -1 & 0 & 1 & & 1 & 1 & 0 \\
\hline$F_{3}(8, n)$ & -1 & 0 & 0 & 1 & 1 & 1 & & 0 & 0 & 0 \\
\hline$n$ & 9 & 8 & 7 & 6 & 5 & 4 & 3 & 2 & 1 & 0 \\
\hline$F_{3}(1, n)$ & 1 & 1 & -1 & 0 & 1 & 0 & 0 & 1 & 1 & 1 \\
\hline$F_{3}(2, n)$ & 1 & -1 & 1 & 0 & 0 & 1 & 0 & 1 & 1 & 1 \\
\hline$F_{3}(3, n)$ & $\frac{1}{8}$ & $\frac{1}{4}$ & $\frac{1}{4}$ & $\frac{1}{4}$ & $\frac{1}{2}$ & $\frac{1}{2}$ & $\frac{1}{2}$ & 1 & 1 & 1 \\
\hline$F_{3}(4, n)$ & 2 & -1 & 1 & 0 & 1 & 0 & 1 & 1 & 1 & 1 \\
\hline$F_{3}(5, n)$ & 0 & 1 & 1 & 0 & 0 & 1 & 1 & 1 & 1 & 1 \\
\hline$F_{3}(6, n)$ & 1 & 0 & 0 & 0 & 1 & 1 & 1 & 1 & 1 & 1 \\
\hline$F_{3}(7, n)$ & 0 & 0 & 0 & 1 & 1 & 1 & 1 & 1 & 1 & 1 \\
\hline$F_{3}(8, n)$ & 0 & 0 & 1 & 1 & 1 & 1 & 1 & 1 & 1 & 1 \\
\hline
\end{tabular}

$F_{3}(3,-n)=\frac{1}{2} F_{3}(3,-n+3)$.

The Table 2 includes the first few elements of $F_{3}(k,-n)$ for special $k$ and negative $n$.

\section{Combinatorial interpretation}

First we give a graph interpretation of $F_{3}(k, n)$ for integer $k \geq 4$. Let $P_{n}, n \geq 2$, be a path without loops and multiple edges with the vertex set $V\left(P_{n}\right)=\left\{v_{1}, \ldots, v_{n}\right\}$. Vertices of $P_{n}$ are numbered in the natural fashion. Let consider a colouring $c$ of vertices of $P_{n}$ such that vertices $v_{n}$ and $v_{n-1}$ can be uncoloured if $k \geq 3$ or the vertex $v_{n}$ can be uncoloured if $k=2$ or all vertices are coloured if $k=1$. Let $\{0,1\}$ be a set of colours and $c\left(v_{j}\right)=i$ denote that the vertex $v_{j}$ has a colour $i, 0 \leq i \leq 1$.

The following recurrent procedure defines the colouring $c$ of $P_{n}$.

Denote by $A$ a set of uncoloured vertices of a path $P_{n}$ and let $m=\min \{3, k\}$.

Until $|A|<m$, repeat following operations:

Let $v_{j} \in A$ be a vertex with the smallest index.

If $|A|=m$, then $c\left(v_{j}\right)=0$, otherwise $c\left(v_{j}\right)=i, 0 \leq i \leq 1$.

If $c\left(v_{j}\right)=0$, then $c\left(v_{j+i}\right)=0$ for $i=1, \ldots, m-1$.

If $c\left(v_{j}\right)=1$, then $c\left(v_{j+i}\right)=1$ for $i=1, \ldots, \max \{3, k\}-1$. 
We can interpret obtained monochromatic paths of the length $k$ or 3 as monochromatic scraps, so defined the above colouring $c$ of $P_{n}$ we will call a $\left\{P_{3}, P_{k}\right\}$-scrap colouring of $P_{n}$. Denote by $P_{3}$ a scrap of three vertices coloured 0 and by $P_{k}$ a scrap of $k$ vertices coloured 1 . In other words the $\left\{P_{3}, P_{k}\right\}$-scrap colouring of $P_{n}$ is a covering of $P_{n}$ by scraps from the set $\left\{P_{3}, P_{k}\right\}$.

Note that for $k=3$ we have two monochromatic scraps of the length 3 with different colours. To distinguish them we will write $P_{3}$ and $P_{3}^{\prime}$.

Consider for example the path $P_{15}$ and $k=5$. Then we have the following possibilities of a $\left\{P_{3}, P_{5}\right\}$-scrap colouring:

(a) $P_{5} P_{5} P_{5}$ and $P_{3} P_{3} P_{3} P_{3} P_{3}$. So we have exactly two colouring.

(b) Colourings when the last vertex is uncoloured are $P_{5} P_{3} P_{3} P_{3}, P_{3} P_{5} P_{3} P_{3}$, $P_{3} P_{3} P_{5} P_{3}, P_{3} P_{3} P_{3} P_{5}$, what gives 4 .

(c) Colourings when the last two vertices are uncoloured are $P_{5} P_{5} P_{3}, P_{5} P_{3} P_{5}$, $P_{3} P_{5} P_{5}$, so there are 3 such colourings.

Summing up, we have that there exist $9\left\{P_{3}, P_{k}\right\}$-scrap colourings of $P_{15}$.

Denote by $\alpha(n)$ the total number of $\left\{P_{3}, P_{k}\right\}$-scrap colourings of $P_{n}$.

Theorem 1 Let $k \geq 1, n \geq 0$ be integers. The number of all $\left\{P_{3}, P_{k}\right\}$-scrap colourings of $P_{n}$ is equal to $F_{3}(k, n)$.

Proof Denote by $\alpha_{k}(n)$ a $\left\{P_{3}, P_{k}\right\}$-scrap colouring starting from $P_{k}$ and by $\alpha_{3}(n)$ a $\left\{P_{3}, P_{k}\right\}$-scrap colouring starting from $P_{3}$. Consider three cases.

Case 1. $k=1$, then all vertices are coloured.

For $n=0$ we take $F_{3}(1,0)=1$, there is no path with 0 vertices.

For $n=1$ we have $F_{3}(1,1)=1$. There is exactly one vertex and exactly one colouring with scrap $P_{1}$.

Analogously for $n=2, F_{3}(1,2)=1$. The unique colouring is $P_{1} P_{1}$.

For $n=3$ we have $F_{3}(1,3)=2$. There are colourings $P_{1} P_{1} P_{1}$ and $P_{3}$.

Assume that $n>3$ and $\alpha(n)=F_{3}(1, n)$. Then

$$
\begin{aligned}
\alpha(n+1) & =\alpha_{3}(n+1)+\alpha_{1}(n+1)=\alpha(n+1-3)+\alpha(n+1-1) \\
& =F_{3}(1, n+1-3)+F_{3}(1, n+1-1)=F_{3}(1, n+1),
\end{aligned}
$$

Case 2. $k=2$, then the vertex $v_{n}$ can be uncoloured.

For $n=0$ we take $F_{3}(2,0)=1$.

$F_{3}(2,1)=1$, the vertex $v_{1}$ is uncoloured.

$F_{3}(2,2)=1$, there is exactly one colouring with scrap $P_{2}$.

$F_{3}(2,3)=2$, there are colourings $P_{2}$ with the vertex $v_{3}$ uncoloured and $P_{3}$.

For $n>3$ we prove analogously as for $k=1$.

Case 3. $k=3$, then vertices $v_{n}$ and $v_{n-1}$ can be uncoloured.

If $n=0$, then we put $F_{3}(3,0)=1$. If $n=1$, then the path consist of one vertex, we leave it uncoloured. So $F_{3}(3,1)=1$. Analogously for $n=2, F_{3}(3,2)=1$.

If $n=3$, then there are two colourings $P_{3}$ and $P_{3}^{\prime}, F_{3}(3,3)=2$. Assume that $n>3$ and $\alpha(n)=F_{3}(3, n)$. Then 


$$
\begin{aligned}
\alpha(n+1) & =\alpha_{3}(n+1)+\alpha_{3}(n+1)=\alpha(n+1-3)+\alpha(n+1-3) \\
& =F_{3}(3, n+1-3)+F_{3}(3, n+1-3)=F_{3}(3, n+1),
\end{aligned}
$$

Case 4. $k>3$, then vertices $v_{n}$ and $v_{n-1}$ can be uncoloured.

If $n=0$, then we put $F_{3}(k, 0)=1$, If $n=1$, then the path consist of one vertex, we leave it uncoloured. So $F_{3}(k, 1)=1$. Analogously for $n=2, F_{3}(k, 2)=1$.

If $3 \leq n \leq k-1$, then there is exactly one $\left\{P_{3}, P_{k}\right\}$-scrap colouring only by scraps $P_{3}$. Thus $\alpha(n)=\alpha_{3}(n)=1=F_{3}(k, n)$.

Assume that $n \geq k$ and $\alpha(n)=F_{3}(k, n)$. Then

$$
\begin{aligned}
\alpha(n+1) & =\alpha_{3}(n+1)+\alpha_{k}(n+1)=\alpha(n+1-3)+\alpha(n+1-k) \\
& =F_{3}(k, n+1-3)+F_{3}(k, n+1-k)=F_{3}(k, n+1),
\end{aligned}
$$

which ends the proof.

From the above graph interpretation it follow direct formulas for $F_{3}(k, n)$.

Theorem 2 Let $k \geq 1, n \geq 0$ be integers. Then

$$
F_{3}(k, n+t)=\sum_{i=0}^{\left\lfloor\frac{n}{k}\right\rfloor}\left(i+\left\lfloor\frac{n-i k}{3}\right\rfloor\right) \text { where } t= \begin{cases}2 & \text { for } k=1 \\ 1 & \text { for } k=2 \\ 0 & \text { for } k \geq 3\end{cases}
$$

Proof If $n \leq k-1$, then $\left\lfloor\frac{n}{k}\right\rfloor=0$ and

$$
F_{3}(k, n)=\sum_{i=0}^{0}\left(\begin{array}{c}
i+\left\lfloor\frac{n-i k}{3}\right\rfloor \\
i
\end{array}\right)=\left(\begin{array}{c}
0+\left\lfloor\frac{n}{3}\right\rfloor \\
0
\end{array}\right)=1
$$

Assume that $n \geq k$. By Theorem 1 , the number $F_{3}(k, n)$ is equal to the number of $\left\{P_{3}, P_{k}\right\}$-scrap colourings of $P_{n}$. Each $\left\{P_{3}, P_{k}\right\}$-scrap colouring consists of $i$ monochromatic paths $P_{k}$ and $j$ monochromatic paths $P_{3}$, where $0 \leq i \leq\left\lfloor\frac{n}{k}\right\rfloor$, $0 \leq j \leq\left\lfloor\frac{n}{k}\right\rfloor$. Moreover, for a fixed $i$ we have $j=\left\lfloor\frac{n-i k}{3}\right\rfloor$ and the number of $\left\{P_{3}, P_{k}\right\}-$ scrap colourings is equal to $\left(\begin{array}{c}i+j \\ i\end{array}\right)=\left(i+\left\lfloor\frac{n-i k}{3}\right\rfloor\right)$. Thus $F_{3}(k, n)=\sum_{i=0}^{\left\lfloor\frac{n}{k}\right\rfloor}\left(i+\left\lfloor\frac{n-i k}{3}\right\rfloor\right)$.

Now we can give an interpretation of $F_{3}(k, n)$ numbers for $k \neq 3$.

Let $k \geq 4, n \geq 3$ be integers, $X=\{1,2,3, \ldots, n\}$, and $\mathcal{Y}=\left\{Y_{t}: t \in T\right\}$ be the family of disjoint subsets of the set $X$ such that each subset $Y_{t}$ contains consecutive integers and satisfies the following conditions.

1. $\left|Y_{t}\right| \in\{3, k\}$,

2. $\left|X \backslash \bigcup_{t \in T} Y_{t}\right| \in\{0,1,2\}$,

3. If $p \in X \backslash \bigcup_{t \in T} Y_{t}$, then $p=n$ or 
4. if $p, q \in X \backslash \bigcup_{t \in T} Y_{t}$, then $p=n$ and $q=n-1$.

The family $\mathcal{Y}$ is called the $(3, k)$-decomposition with the rest or a $(3, k)$ decomposition family.

If $X \backslash \bigcup_{t \in T} Y_{t}=\emptyset$, then we have a decomposition of the set $X$.

Theorem 3 Let $k \geq 1, k \neq 3, n \geq 3$ be integers. Then the number of all $(3, k)$ decompositions with the rest at most two of the set $X$ is equal to $F_{3}(k, n)$.

Proof We will prove for $k \geq 4, n \geq 3$. For $k=1,2$ we prove analogously. Denote by $d(n)$ the number of all $(3, k)$-decompositions of the set $X$. If $n=3$, then we have the only one decomposition $\{\{1,2,3\}\}$. Thus $d(3)=1=F_{3}(k, 3)$. Analogously for $3<n \leq k-1$ by inspections the result follows.

Let $n \geq k$ and suppose that the equality $d(n)=F_{3}(k, n)$ holds for an arbitrary $n$. We will show that $d(n+1)=F_{3}(k, n+1)$.

Denote by $d_{3}(n+1)$ the number of all $(3, k)$-decompositions of $X=$ $\{1,2, \ldots, n+1\}$ such that $Y=\{1,2,3\}$, and let $d_{k}(n+1)$ be the number of all (3, $k$ )-decompositions of $X=\{1,2, \ldots, n+1\}$ such that $Y=\{1,2, \ldots, k\}$. We have that $d(n+1)=d_{3}(n+1)+d_{k}(n+1) \quad$ and $\quad d_{3}(n+1)=d(n+1-3)$, $d_{k}(n+1)=d(n+1-k)$. By the induction hypothesis and the recurrence (5) we have

$$
\begin{aligned}
d_{3}(n+1) & =d(n+1-3)+ \\
d(n+1-k) & =F_{3}(k, n+1-3)+F_{3}(k, n+1-k)=F_{3}(k, n+1),
\end{aligned}
$$

which ends the proof.

The above interpretation of $F_{3}(k, n)$ leads to another interpretation of it in terms of ordered composition, with the rest at most two, of an integer. We have that $|X|=n$ and each subset $Y_{t}$, containing consecutive integers, corresponds to the addend $n_{t}$. A sum $\sum_{t \in T} n_{t}+n_{r}=n$ we call an ordered compositions, with the rest, of an integer $n$ if

1. $n_{t} \in\{3, k\}$,

2. $n_{r} \in\{0,1,2\}$,

3. $\sum_{t \in T} n_{t}+n_{r}=n$ and $n_{r}$ is the last addend in the sum.

\section{Generating function and some identities}

Theorem 4 Let $n \geq 0, k \geq 1$ be integers. The generating function of $F_{3}(k, n)$ has the following form

$$
g(x)=\frac{1+t}{1-x^{3}-x^{k}} \text { where } t=\left\{\begin{array}{c}
0 \text { for } k=1 \\
x \text { for } k=2 \\
x+x^{2} \text { for } k \geq 3
\end{array} .\right.
$$


Proof Let $g(x)=\sum_{n=0}^{\infty} F_{3}(k, n) x^{n}$. Using the recurrence (5) we have $g(x)-$ $x^{3} g(x)-x^{k} g(x)=1+t$ where $t=\left\{\begin{array}{c}0 \text { for } k=1 \\ x \text { for } k=2 \\ x+x^{2} \text { for } k \geq 3\end{array}\right.$ Hence $g(x)=\frac{1+t}{1-x^{3}-x^{k}}$ which ends the proof.

From the Theorem 4, for special values of $k$, we obtain generating functions for Narayana numbers and Padovan numbers.

Corollary 5 If $k=1$, then $g(x)=\frac{1}{1-x-x^{3}}$ is the generating functions of Narayana numbers [14].

If $k=2$, then $g(x)=\frac{1+x}{1-x^{2}-x^{3}}$ is the generating functions of Padovan numbers.

The next theorem proofs that all $F_{3}(3 k, n)$ sequences are tripled.

Theorem 6 Let $k \geq 1, n \geq 0$ be integers. Then

$$
F_{3}(3 k, 3 n)=F_{3}(3 k, 3 n+1)=F_{3}(3 k, 3 n+2) .
$$

Proof by induction on $\boldsymbol{n}$ From the definition of $F_{3}(k, n)$ we have that $F_{3}(3 k, 0)=\cdots=F_{3}(3 k, 3 k-1)=1$. By the formula $(5), \quad F_{3}(3 k, 3 k)=$ $F_{3}(3 k, 3 k+1)=F_{3}(3 k, 3 k+2)=2$ and next $F_{3}(3 k, 3 k+3)=F_{3}(3 k, 3 k+4)=$ $F_{3}(3 k, 3 k+5)=3$.

Assume that $F_{3}(3 k, 3 t)=F_{3}(3 k, 3 t+1)=F_{3}(3 k, 3 t+2)$ for all $t \leq n$.

We will prove

$$
F_{3}(3 k, 3(n+1))=F_{3}(3 k, 3(n+1)+1)=F_{3}(3 k, 3(n+1)+2) .
$$

For consecutive numbers we have

$$
F_{3}(3 k, 3(n+1))=F_{3}(3 k, 3 n+3)
$$

$=F_{3}(3 k, 3 n)+F_{3}(3 k, 3 n+3-3 k)=F_{3}(3 k, 3 n)+F_{3}(3 k, 3(n+1-k))$,

$F_{3}(3 k, 3(n+1)+1)=F_{3}(3 k, 3 n+4)=F_{3}(3 k, 3 n+1)+F_{3}(3 k, 3 n+4-3 k)$ $=F_{3}(3 k, 3 n+1)+F_{3}(3 k, 3(n+1-k)+1)$,

$F_{3}(3 k, 3(n+1)+2)=F_{3}(3 k, 3 n+5)=F_{3}(3 k, 3 n+2)+F_{3}(3 k, 3 n+5-3 k)$ $=F_{3}(3 k, 3 n+2)+F_{3}(3 k, 3(n+1-k)+2)$.

Using our assumption we ascertain that the above numbers are equal.

Theorem 7 Let $k \geq 3, n \geq 0$ be integers. Then

$$
\sum_{i=0}^{n} F_{3}(k, 3 i+t)=F_{3}(k, 3 n+k+t)-1, \text { for } 0 \leq t \leq 2
$$

Proof by induction on $n$ We will prove for an arbitrary $k \geq 3$ and $t=0$. For $k \geq 3$, $t=1$ and $t=2$ we prove analogously.

If $n=0$, then $F_{3}(k, 0)=1=F_{3}(k, 3 \cdot 0+k)-1=2-1=1$.

If $n=1$, then for $k=3$ we have $F_{3}(k, 0)+F_{3}(k, 3)=1+2=F_{3}(k, 3+3)-$ 
$1=4-1=3$ and

for $k>3, F_{3}(k, 0)+F_{3}(k, 3)=1+1=F_{3}(k, 3+k)-1=3-1=2$.

Assume that

$$
\sum_{i=0}^{n} F_{3}(k, 3 i)=F_{3}(k, 3 n+k)-1
$$

Then

$$
\begin{aligned}
\sum_{i=0}^{n+1} F_{3}(k, 3 i)= & \sum_{i=0}^{n} F_{3}(k, 3 i)+F_{3}(k, 3(n+1)) \\
& =F_{3}(k, 3 n+k)-1+F_{3}(k, 3 n+3)=F_{3}(k, 3(n+1)+k)-1 .
\end{aligned}
$$

Thus the theorem is proved.

\section{Matrix generators}

Let $Q_{k}=\left[q_{i j}\right]_{k \times k}$ be a square matrix. For a fixed $1 \leq i \leq k$ an element $q_{i 1}$ is equal to the coefficient at $F_{3}(k, i)$ of the right hand side of the formula (5). For $j>1$ and an arbitrary $i$ we have $q_{i j}=1$ if $j=i+1$ and $q_{i j}=0$, otherwise.

The above definition gives matrices

$$
Q_{3}=\left[\begin{array}{lll}
0 & 1 & 0 \\
0 & 0 & 1 \\
2 & 0 & 0
\end{array}\right], Q_{4}=\left[\begin{array}{cccc}
0 & 1 & 0 & 0 \\
0 & 0 & 1 & 0 \\
1 & 0 & 0 & 1 \\
1 & 0 & 0 & 0
\end{array}\right], \ldots, Q_{k}=\left[\begin{array}{ccccc}
0 & 1 & 0 & \cdots & 0 \\
0 & 0 & 1 & \cdots & 0 \\
1 & 0 & 0 & \cdots & 0 \\
\vdots & \vdots & \vdots & \ddots & \vdots \\
0 & 0 & 0 & \cdots & 1 \\
1 & 0 & 0 & \cdots & 0
\end{array}\right] .
$$

Moreover, we define a square matrix $P_{k}$ of order $k$ as the matrix of initial conditions

$$
P_{k}=\left[\begin{array}{ccccc}
F_{3}(k, 2 k-2) & F_{3}(k, 2 k-3) & \cdots & F_{3}(k, k) & F_{3}(k, k-1) \\
F_{3}(k, 2 k-3) & F_{3}(k, 2 k-4) & \cdots & F_{3}(k, k-1) & F_{3}(k, k-2) \\
\vdots & \vdots & \ddots & \vdots & \vdots \\
F_{3}(k, k) & F_{3}(k, k-1) & \cdots & F_{3}(k, 2) & F_{3}(k, 1) \\
F_{3}(k, k-1) & F_{3}(k, k-2) & \cdots & F_{3}(k, 1) & F_{3}(k, 0)
\end{array}\right] .
$$

Using Laplace'a Theorem and basic properties of determinants, we get the following results.

Theorem 8 Let $k \geq 3$ be an integer. Then 


$$
\begin{array}{r}
\operatorname{det} Q_{3}=2 \text { and } \operatorname{det} Q_{k}=(-1)^{k+1}, \\
\operatorname{det} P_{k}=(-1)^{2 k+\frac{k(k+1)}{2}-3} .
\end{array}
$$

Theorem 9 Let $k \geq 3, n \geq 1$ be integers. Then

$$
P_{k} Q_{k}^{n}=\left[\begin{array}{ccccc}
F_{3}(k, n+2 k-2) & F_{3}(k, n+2 k-3) & \cdots & F_{3}(k, n+k) & F_{3}(k, n+k-1) \\
F_{3}(k, n+2 k-3) & F_{3}(k, n+2 k-4) & \cdots & F_{3}(k, n+k-1) & F_{3}(k, n+k-2) \\
\vdots & \vdots & \ddots & \vdots & \vdots \\
F_{3}(k, n+k) & F_{3}(k, n+k-1) & \cdots & F_{3}(k, n+2) & F_{3}(k, n+1) \\
F_{3}(k, n+k-1) & F_{3}(k, n+k-2) & \cdots & F_{3}(k, n+1) & F_{3}(k, n)
\end{array}\right] .
$$

Proof If $n=1$, then by (5) and simple calculations the result immediately follows. Assume the formula (9) holds for $n$, we will prove it for $n+1$. Since $P_{k} Q_{k}^{n+1}=\left(P_{k} Q_{k}^{n}\right) Q_{k}$, by our assumption and by the recurrence (5) we obtain

$$
\begin{aligned}
A_{k} Q_{k}^{n+1}= & {\left[\begin{array}{ccccc}
F_{3}(k, n+2 k-2) & F_{3}(k, n+2 k-3) & \cdots & F_{3}(k, n+k) & F_{3}(k, n+k-1) \\
F_{3}(k, n+2 k-3) & F_{3}(k, n+2 k-4) & \cdots & F_{3}(k, n+k-1) & F_{3}(k, n+k-2) \\
\vdots & \vdots & \ddots & \vdots & \vdots \\
F_{3}(k, n+k) & F_{3}(k, n+k-1) & \cdots & F_{3}(k, n+2) & F_{3}(k, n+1) \\
F_{3}(k, n+k-1) & F_{3}(k, n+k-2) & \cdots & F_{3}(k, n+1) & F_{3}(k, n)
\end{array}\right] } \\
& {\left[\begin{array}{ccccc}
0 & 1 & 0 & \cdots & 0 \\
0 & 0 & 1 & \cdots & 0 \\
1 & 0 & 0 & \cdots & 0 \\
\vdots & \vdots & \vdots & \ddots & \vdots \\
0 & 0 & 0 & \cdots & 1 \\
1 & 0 & 0 & \cdots & 0
\end{array}\right] } \\
& {\left[\begin{array}{cccccc}
F_{3}(k, n+2 k-1) & F_{3}(k, n+2 k-2) & \cdots & F_{3}(k, n+k+1) & F_{3}(k, n+k) \\
F_{3}(k, n+2 k-2) & F_{3}(k, n+2 k-3) & \cdots & F_{3}(k, n+k) & F_{3}(k, n+k-1) \\
\vdots & \vdots & \ddots & & \\
F_{3}(k, n+k+1) & F_{3}(k, n+k) & \cdots & F_{3}(k, n+3) & F_{3}(k, n+2) \\
F_{3}(k, n+k) & F_{3}(k, n+k-1) & \cdots & F_{3}(k, n+2) & F_{3}(k, n+1)
\end{array}\right], }
\end{aligned}
$$

which ends the proof.

By Theorem 8 we get the following result.

Corollary 10 Let $k \geq 3, n \geq 2$ be integers. Then 
Table 3 Distance Fibonacci numbers $F_{3}^{i}(5, n)$ and $F_{3}(5, n)$

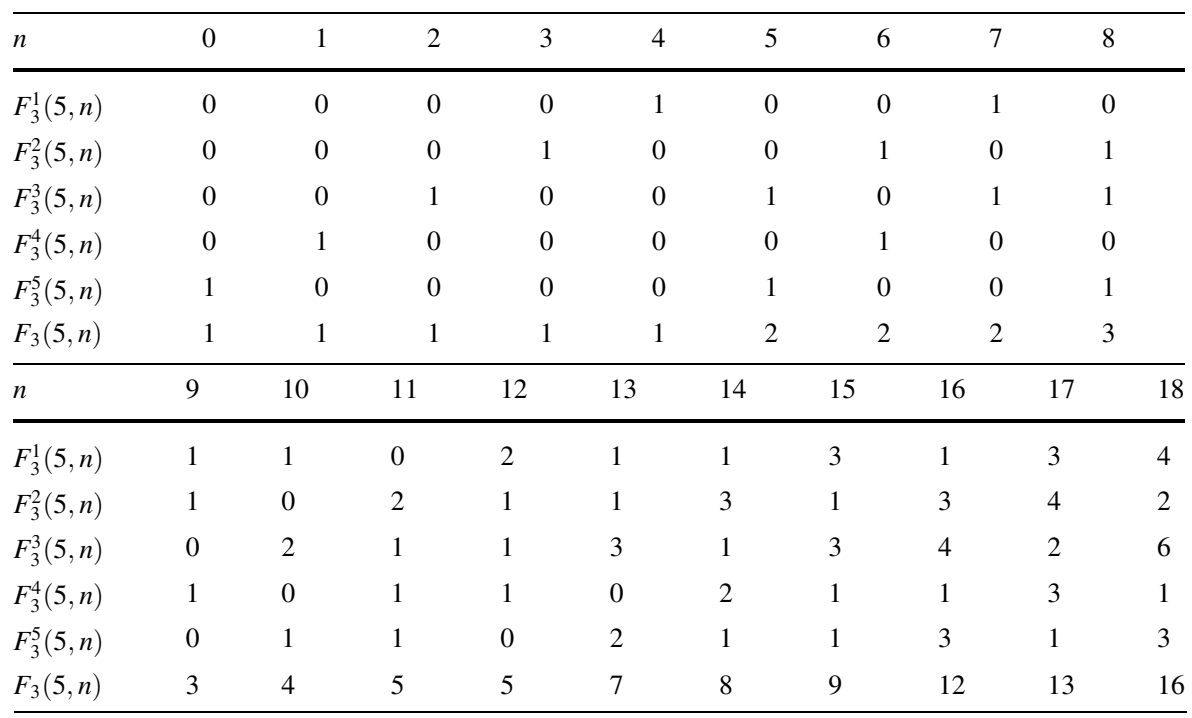

$$
\operatorname{det} P_{k} Q_{k}^{n}=(-1)^{\left[2 k+\frac{k(k+1)}{2}-3\right]+n(k+1)} \text {. }
$$

\section{Connections with the Pascal's triangle}

To study connections of $(3, k)$-distance Fibonacci numbers with Pascal's triangle we need to consider a family of sequences given by the same recurrence as $F_{3}(k, n)$ with different initial conditions.

Let $k \geq 1, n \geq 0$ be integers and

$$
F_{3}^{i}(k, n)=F_{3}^{i}(k, n-3)+F_{3}^{i}(k, n-k)
$$

with $F_{3}^{i}(k, n)=\left\{\begin{array}{c}1 \text { if } n=k-i \\ 0 \text { in othervise }\end{array}\right.$ for $n=0,1, \ldots, \max \{2, k\}$.

A few initial elements of these sequences for $k=5$ and special values $n$ are included in the Table 3 .

Based on results from [16] we have

Theorem 11 Let $k \geq 4, n \geq 0,0 \leq i \leq k-1$ be integers. Then

$$
F_{3}(k, n)=\sum_{i=0}^{\max \{2, k-1\}} F_{3}^{i}(k, n) .
$$


Fig. 2 Digraph $D$

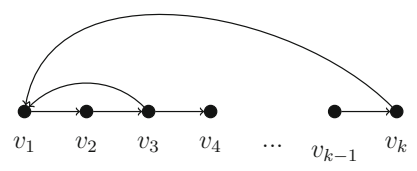

Er shoved [3] that $n$th power of the companion matrix $Q_{k}$ contains entries of sequences $F_{3}^{i}(k, n)$,

$$
Q_{k}^{n}=\left[\begin{array}{cccc}
F_{3}^{1}(k, n+k-1) & F_{3}^{1}(k, n+k-2) & \ldots & F_{3}^{1}(k, n) \\
F_{3}^{2}(k, n+k-1) & F_{3}^{2}(k, n+k-2) & \ldots & F_{3}^{2}(k, n) \\
\vdots & \vdots & \ddots & \vdots \\
F_{3}^{k}(k, n+k-1) & F_{3}^{k}(k, n+k-2) & \ldots & F_{3}^{k}(k, n)
\end{array}\right] .
$$

The matrix $Q_{k}$ we can interpret as the adjacency matrix of a special digraph $D$, see the Fig. 2.

It is well known that $Q_{k}^{n}$ contains the number off all different paths of length $n$ between corresponding vertices in the digraph $D$. Namely, the entry $q_{i j}$ is equal to the number of all paths of the length $n$ from vertex $v_{i}$ to vertex $v_{j}$ in the digraph $D$.

Using such interpretation we can prove

Theorem 12 Let $k \geq 4, n \geq 0,0 \leq i \leq k-1$ be integers. Then

$$
\begin{aligned}
& F_{3}^{1}(k, n)=\sum_{\alpha_{3}, \alpha_{k}}\left(\begin{array}{c}
\alpha_{3}+\alpha_{k} \\
\alpha_{3}
\end{array}\right) \\
& 3 \alpha_{3}+k \alpha_{k}=n \\
& F_{3}^{j}(k, n)=\quad \sum_{\alpha_{3}, \alpha_{k}} \quad\left(\begin{array}{c}
\alpha_{3}+\alpha_{k} \\
\alpha_{3}
\end{array}\right) \\
& 3 \alpha_{3}+k \alpha_{k}=n-(3-j+1) \\
& +\quad \sum_{\alpha_{3}, \alpha_{k}} \quad\left(\begin{array}{c}
\alpha_{3}+\alpha_{k} \\
\alpha_{3}
\end{array}\right) \text { for } j=2,3 \text {, } \\
& 3 \alpha_{3}+k \alpha_{k}=n-(k-j+1) \\
& F_{3}^{j}(k, n)=\quad \sum_{\alpha_{3}, \alpha_{k}} \quad\left(\begin{array}{c}
\alpha_{3}+\alpha_{k} \\
\alpha_{3}
\end{array}\right) \text { for } 3<j \leq k . \\
& 3 \alpha_{3}+k \alpha_{k}=n-(k-j+1)
\end{aligned}
$$

Proof For a fixed $1 \leq j \leq k$ the element $q_{j, 1}$ of $Q_{k}^{n}$ is equal to the total number of different directed paths of length $n$ from the vertex $v_{j}$ to the vertex $v_{1}$ in digraph $D$, see the Fig. 2. Each such path contains at the beginning a short path $P=$ 
$v_{j}-\cdots-v_{1}$ and next a sequence $\mathcal{C}$ of cycles of length 3 or $k$.

Let consider a number of such paths of length $n$ with dependence of short path $P$.

Case 1. $P=v_{1}-v_{2}-v_{3}-v_{1}$ or $P=v_{1}-v_{2}-v_{3}-v_{4}-\cdots-v_{k}-v_{1}$. In this case a path $P$ form a cycle $C_{3}$ or $C_{k}$, respectively. The whole path is a sequence of cycles $C_{3}$ or $C_{k}$ in random order; its length $n=3 \alpha_{3}+k \alpha_{k}$ for all $\alpha_{3}, \alpha_{k}$ satisfying the equality. Thus we have

$$
\sum_{\substack{\alpha_{3}, \alpha_{k} \\
3 \alpha_{3}+k \alpha_{k}=n}}\left(\begin{array}{c}
\alpha_{3}+\alpha_{k} \\
\alpha_{3}
\end{array}\right)=F_{3}^{1}(k, n)
$$

such paths.

Case 2. $P=v_{j}-v_{j+1}-\cdots-v_{k}-v_{1}, 1<j \leq k$.

The path $P$ has a length $k-j+1$ and remaining part of length $n-(k-j+1)=$ $3 \alpha_{3}+k \alpha_{k}$ consists of cycles $C_{3}$ or $C_{k}$. Analogously as in the Case 1 we have

$$
\sum_{\alpha_{3}, \alpha_{k}}\left(\begin{array}{c}
\alpha_{3}+\alpha_{k} \\
\alpha_{3}
\end{array}\right)
$$

such paths.

Case 3. $P=v_{j} \cdots-v_{3}-v_{1}, 1<j \leq 3$.

The path $P$ has length $3-j+1$, the remaining part has the length $n-(3-j+1)=3 \alpha_{3}+k \alpha_{k}$, and there is exactly

$$
\sum_{\alpha_{3}, \alpha_{k}}\left(\begin{array}{c}
\alpha_{3}+\alpha_{k} \\
\alpha_{3}
\end{array}\right)
$$

such paths.

Note that for $j=2,3$ we have

$$
\begin{gathered}
\sum_{\alpha_{3}, \alpha_{k}}\left(\begin{array}{c}
\alpha_{3}+\alpha_{k} \\
\alpha_{3}
\end{array}\right)+\sum_{\alpha_{3}, \alpha_{k}} \\
3 \alpha_{3}+ \\
3 \alpha_{3}+k \alpha_{k}=n-(3-j+1) \\
k \alpha_{k}=n-(k-j+1)\left(\begin{array}{c}
\alpha_{3}+\alpha_{k} \\
\alpha_{3}
\end{array}\right)=F_{3}^{j}(k, n) \text {.For } 3<j \leq k \text { we have } \\
\sum_{\alpha_{3}, \alpha_{k}}\left(\begin{array}{c}
\alpha_{3}+\alpha_{k} \\
\alpha_{3}
\end{array}\right)=F_{3}^{j}(k, n) . \\
3 \alpha_{3}+k \alpha_{k}=n-(k-j+1)
\end{gathered}
$$


Based on the Theorem 11 and the Theorem 12 we have

Theorem 13 Let $k \geq 4, n \geq 0$ be integers. Then

$$
\begin{gathered}
F_{3}(k, n)=\sum_{i=0}^{2} \sum_{\alpha_{3}, \alpha_{k}}\left(\begin{array}{c}
\alpha_{3}+\alpha_{k} \\
\alpha_{3}
\end{array}\right) \\
+\sum_{i=0}^{k-1} \sum_{\substack{\alpha_{3}+k \alpha_{k}=n-(3-i+1) \\
3 \alpha_{3}+k \alpha_{k}=n-(k-i+1)}}\left(\begin{array}{c}
\alpha_{3}+\alpha_{k} \\
\alpha_{3}
\end{array}\right) .
\end{gathered}
$$

From the Theorem 13 we can obtain binomials whose sums are equal to numbers $F_{3}(k, n)$. Using these binomials we can derive new formulas for $F_{3}(k, n)$ numbers. For a convenience we use a graphical presentation.

For example, the number $F_{3}(4,25)$ is a sum of

$$
\begin{aligned}
& \left(\begin{array}{l}
8 \\
7
\end{array}\right),\left(\begin{array}{l}
7 \\
3
\end{array}\right),\left(\begin{array}{l}
8 \\
8
\end{array}\right),\left(\begin{array}{l}
7 \\
4
\end{array}\right),\left(\begin{array}{l}
6 \\
0
\end{array}\right),\left(\begin{array}{l}
7 \\
5
\end{array}\right),\left(\begin{array}{l}
6 \\
1
\end{array}\right),\left(\begin{array}{l}
7 \\
6
\end{array}\right),\left(\begin{array}{l}
6 \\
2
\end{array}\right) \text { and }\left(\begin{array}{l}
8 \\
8
\end{array}\right), \\
& \left(\begin{array}{l}
7 \\
4
\end{array}\right),\left(\begin{array}{l}
6 \\
0
\end{array}\right),\left(\begin{array}{l}
7 \\
5
\end{array}\right),\left(\begin{array}{l}
6 \\
1
\end{array}\right) ; \text { and } F_{3}(4,26) \text { is a sum of }\left(\begin{array}{l}
8 \\
6
\end{array}\right),\left(\begin{array}{l}
7 \\
2
\end{array}\right),
\end{aligned}
$$$$
\left(\begin{array}{l}
8 \\
7
\end{array}\right),\left(\begin{array}{l}
7 \\
3
\end{array}\right),\left(\begin{array}{l}
8 \\
8
\end{array}\right),\left(\begin{array}{l}
7 \\
4
\end{array}\right),\left(\begin{array}{l}
6 \\
0
\end{array}\right),\left(\begin{array}{l}
7 \\
5
\end{array}\right)\left(\begin{array}{l}
6 \\
1
\end{array}\right) \text { and }\left(\begin{array}{l}
8 \\
7
\end{array}\right),\left(\begin{array}{l}
7 \\
3
\end{array}\right),\left(\begin{array}{l}
8 \\
8
\end{array}\right),\left(\begin{array}{l}
7 \\
4
\end{array}\right),\left(\begin{array}{l}
6 \\
0
\end{array}\right) \text {. }
$$

These binomials form a geometrical pattern, we will call it a staircase, useful for calculating $F_{3}(4, n)$ numbers from the Pascal Triangle.

$$
\left[\begin{array}{lllllllllll}
1 & 0 & 0 & 0 & 0 & 0 & 0 & 0 & 0 & 0 & 0 \\
1 & 1 & 0 & 0 & 0 & 0 & 0 & 0 & 0 & 0 & 0 \\
1 & 2 & 1 & 0 & 0 & 0 & 0 & 0 & 0 & 0 & 0 \\
1 & 3 & 3 & 1 & 0 & 0 & 0 & 0 & 0 & 0 & 0 \\
1 & 4 & 6 & 4 & 1 & 0 & 0 & 0 & 0 & 0 & 0 \\
1 & 5 & 10 & 10 & 5 & 1 & 0 & 0 & 0 & 0 & 0 \\
1 & 6 & 15 & 20 & 15 & 6 & 1 & 0 & 0 & 0 & 0 \\
\hline 1 & 7 & 21 & 35 & 35 & 21 & 7 & 1 & 0 & 0 & 0 \\
1 & 8 & 28 & 56 & 70 & 56 & 28 & 8 & 1 & 0 & 0 \\
1 & 9 & 36 & 84 & 126 & 126 & 84 & 36 & 9 & 1 & 0 \\
1 & 10 & 45 & 120 & 210 & 252 & 210 & 120 & 45 & 10 & 1
\end{array}\right]
$$




$$
\left[\begin{array}{lllllllllll}
1 & 0 & 0 & 0 & 0 & 0 & 0 & 0 & 0 & 0 & 0 \\
1 & 1 & 0 & 0 & 0 & 0 & 0 & 0 & 0 & 0 & 0 \\
1 & 2 & 1 & 0 & 0 & 0 & 0 & 0 & 0 & 0 & 0 \\
1 & 3 & 3 & 1 & 0 & 0 & 0 & 0 & 0 & 0 & 0 \\
1 & 4 & 6 & 4 & 1 & 0 & 0 & 0 & 0 & 0 & 0 \\
1 & 5 & 10 & 10 & 5 & 1 & 0 & 0 & 0 & 0 & 0 \\
1 & 6 & 15 & 20 & 15 & 6 & 1 & 0 & 0 & 0 & 0 \\
\overline{1} & 7 & 21 & 35 & 35 & 21 & 7 & 1 & 0 & 0 & 0 \\
1 & 8 & 28 & 56 & 70 & 56 & 28 & 8 & 1 & 0 & 0 \\
1 & 9 & 36 & 84 & 126 & 126 & 84 & 36 & 9 & 1 & 0 \\
1 & 10 & 45 & 120 & 210 & 252 & 210 & 120 & 45 & 10 & 1
\end{array}\right]
$$

We extending the staircase presented above up to infinity in both directions. By moving such infinite staircase one column to the left, we obtain next number $F_{3}(k, n)$.

In almost each step of the staircase we have two binomials adjacent. Using the basic property of binomials

$$
\left(\begin{array}{l}
n \\
k
\end{array}\right)+\left(\begin{array}{c}
n \\
k+1
\end{array}\right)=\left(\begin{array}{l}
n+1 \\
k+1
\end{array}\right)
$$

we immediately obtain a new simplest staircase.

$$
\left[\begin{array}{lllllllllll}
1 & 0 & 0 & 0 & 0 & 0 & 0 & 0 & 0 & 0 & 0 \\
1 & 1 & 0 & 0 & 0 & 0 & 0 & 0 & 0 & 0 & 0 \\
1 & 2 & 1 & 0 & 0 & 0 & 0 & 0 & 0 & 0 & 0 \\
1 & 3 & 3 & 1 & 0 & 0 & 0 & 0 & 0 & 0 & 0 \\
1 & 4 & 6 & 4 & 1 & 0 & 0 & 0 & 0 & 0 & 0 \\
1 & 5 & 10 & 10 & 5 & 1 & 0 & 0 & 0 & 0 & 0 \\
1 & 6 & 15 & 20 & 15 & 6 & 1 & 0 & 0 & 0 & 0 \\
1 & 7 & 21 & 35 & 35 & 21 & 7 & 1 & 0 & 0 & 0 \\
\hline 1 & 8 & 28 & 56 & 70 & 56 & 28 & 8 & 1 & 0 & 0 \\
1 & 9 & 36 & 84 & 126 & 126 & 84 & 36 & 9 & 1 & 0 \\
1 & 10 & 45 & 120 & 210 & 252 & 210 & 120 & 45 & 10 & 1
\end{array}\right]
$$

Such transformations of the formula from the Theorem 13 leads to

Corollary 14 Let $k \geq 4, n \geq 0$ be integers.

$$
F_{3}(4, n+3)=\sum_{i=0}^{\left\lfloor\frac{n+1}{3}\right\rfloor} \operatorname{sgn}(n+i-2 \bmod 4)\left(\left\lfloor\frac{n+i+1}{4}\right\rfloor\right) .
$$

We obtain a new formula from (15) using (14). Corresponding staircase is presented on the next Pascal's triangle. 


$$
\left[\begin{array}{lllllllllll}
1 & 0 & 0 & 0 & 0 & 0 & 0 & 0 & 0 & 0 & 0 \\
1 & 1 & 0 & 0 & 0 & 0 & 0 & 0 & 0 & 0 & 0 \\
1 & 2 & 1 & 0 & 0 & 0 & 0 & 0 & 0 & 0 & 0 \\
1 & 3 & 3 & 1 & 0 & 0 & 0 & 0 & 0 & 0 & 0 \\
1 & 4 & 6 & 4 & 1 & 0 & 0 & 0 & 0 & 0 & 0 \\
1 & 5 & 10 & 10 & 5 & 1 & 0 & 0 & 0 & 0 & 0 \\
1 & 6 & 15 & 20 & 15 & 6 & 1 & 0 & 0 & 0 & 0 \\
1 & 7 & 21 & 35 & 35 & 21 & 7 & 1 & 0 & 0 & 0 \\
1 & 8 & 28 & 56 & 70 & 56 & 28 & 8 & 1 & 0 & 0 \\
1 & 9 & 36 & 84 & 126 & 126 & 84 & 36 & 9 & 1 & 0 \\
1 & 10 & 45 & 120 & 210 & 252 & 210 & 120 & 45 & 10 & 1
\end{array}\right]
$$

\section{So we can write}

Corollary 15 Let $k \geq 4, n \geq 0$ be integers.

$$
F_{3}(4, n+4)=\sum_{i=0}^{\left\lfloor\frac{n+1}{3}\right\rfloor} \operatorname{sgn}(n+i-2 \bmod 2)\left(\left\lfloor\frac{n+i+1}{4}\right\rfloor\right) .
$$

Open Access This article is licensed under a Creative Commons Attribution-NonCommercial 4.0 International License, which permits any non-commercial use, sharing, adaptation, distribution and reproduction in any medium or format, as long as you give appropriate credit to the original author(s) and the source, provide a link to the Creative Commons licence, and indicate if changes were made. The images or other third party material in this article are included in the article's Creative Commons licence, unless indicated otherwise in a credit line to the material. If material is not included in the article's Creative Commons licence and your intended use is not permitted by statutory regulation or exceeds the permitted use, you will need to obtain permission directly from the copyright holder. To view a copy of this licence, visit http://creativecommons.org/licenses/by-nc/4.0/.

\section{References}

1. Bednarz, U., Włoch, I., Wołowiec-Musiał, M.: Total graph interpretation of numbers of the Fibonacci type. J. Appl. Math. Article ID 837917 (2015)

2. Bednarz, U., Włoch, A., Wołowiec-Musiał, M.: Distance Fibonacci numbers, their interpretations and matrix generators. Commentationes Mathematicae 53(1), 35-46 (2013)

3. Er, M.C.: Sums of Fibonacci numbers by matrix methods. Fibonacci Quart. 22(3), 204-207 (1984)

4. Kiliç, E.: The generalized order-k-Fibonacci-Pell sequence by matrix methods. J. Comput. Appl. Math. 209, 133-145 (2007)

5. Kiliç, E., Tasci, D.: On the generalized Fibonacci and Pell sequences by Hessenberg matrices. Ars Combinatoria 94, 161-174 (2010)

6. Koshy, T.: Fibonacci and Lucas Numbers with Applications. A Wiley-Interscience Publication (2001)

7. Kwaśnik, M., Włoch, I.: The total number of generalized stable sets and kernels in graphs. Ars Combinatoria 55, 139-146 (2000)

8. Miles, E.P., Jr.: Generalized Fibonacci numbers and associated matrices. Am. Math. Mon. 67, 745-752 (1960)

9. Özkan, E., Altun, İ.: Generalized Lucas polynomials and relationships between the Fibonacci polynomials and Lucas polynomials. Commun. Algebra 47, 10-12 (2019) 
10. Özkan, E., Kuloğlu, B.: On the new Narayana polynomials, the Gauss Narayana numbers and their polynomials. Asian-Eur. J. Math. 14(06), 2150100 (2021). https://doi.org/10.1142/ S179355712150100X

11. Özkan, E., Taştan, M.: On Gauss Fibonacci polynomials, Gauss Lucas polynomials and their applications. Commun. Algebra 48(3), 952-960 (2020)

12. Özkan, E., Taştan, M., Aydoğdu, A.: 2-Fibonacci polynomials in the family of Fibonacci numbers. Notes Number Theory Discrete Math. 24, 47-55 (2018)

13. Sloane, N.J.A. (ed.): The on-line encyclopedia of integer sequences. https://oeis.org/. Accessed 10 May 2021

14. Soykan, Y.: On generalized Narayana numbers. Int. J. Adv. Appl. Math. Mech. 3, 43-56 (2020)

15. Włoch, I., Bednarz, U., Bród, D., Włoch, A., Wołowiec-Musiał, M.: On a new type of distance Fibonacci numbers. Discrete Appl. Math. 161, 2695-2701 (2013)

16. Włoch, I., Włoch, A.: On some multinomial sums related to the Fibonacci type numbers. Tatra Mt. Math. Publ. 77, 99-108 (2020)

Publisher's Note Springer Nature remains neutral with regard to jurisdictional claims in published maps and institutional affiliations. 